\title{
Fault Tolerant Control Using Fractional-order Terminal Sliding Mode Control for Robotic Manipulators
}

\author{
Saim AHMED, Haoping WANG*, Yang TIAN \\ Sino-French International Joint Laboratory of Automatic Control and Signal Processing (LaFCAS), \\ School of Automation, Nanjing University of Science \& Technology, Nanjing, 210094, China \\ (*Corresponding author) hp.wang@njust.edu.cn
}

\begin{abstract}
In this paper, Fractional-order Non-singular Fast Terminal Sliding Mode Control based Fault Tolerant Control (FONFTSM-FTC) is investigated for robotic manipulators in the presence of uncertainties, unknown external load disturbances and actuator faults. Firstly, Fractional-order Non-singular Fast Terminal Sliding Mode Control (FONFTSM) is proposed, in which Fractional-order is used to obtain good tracking performance, and Non-singular Fast Terminal Sliding Mode Control is utilized to achieve fast finite-time convergence, non-singularity and chatter-free control inputs. Secondly, FONFTSM with Adaptive scheme is used as a robust Fault Tolerant Control (FTC) to estimate the uncertainties as well as actuator faults without prior knowledge of the upper bounds. Asymptotical stability of the closed-loop system is obtained by Lyapunov analysis. Finally, corresponding simulation results with compared recent methods of adaptive FONFTSM (AFONTSM) and Non-singular Fast Terminal Sliding Mode Control based Active FTC (NFTSMC-FTC) are made to validate and demonstrate the effectiveness of the proposed method.
\end{abstract}

Keywords: Robotic manipulators, Fractional-order, Terminal sliding mode control, Fault tolerant control, Finite-time convergence.

\section{Introduction}

Robotic manipulator is a high nonlinear system, which desires robust stability and trajectory tracking performance in the existence of uncertainties and external disturbances. These unknown uncertainties increase the difficulty to obtain good tracking performance for applied control methods [9]. Moreover, another problem arises when malfunction occurs at the joint actuators. In that case, Fault Tolerant Control (FTC) is used to compensate controller failures for handling the system properly. The control failure is inevitable in the practical situations. Thus, the controlled system cannot work accurately if the controller is not capable to tolerate the faults. Therefore, an increasing interest has been perceived in designing FTC methods, which have been extensively considered and employed in a variety of areas [6]. Basically, the key point behind FTC is that the control system should be reliable to ensure the desired performance and asymptotical stability in the presence of actuator failures [5].

The Sliding Mode Control (SMC) which belongs to the class of a nonlinear controller has the capability to deal efficiently nonlinear systems with uncertainties, bounded external load disturbances, and small sensitivity to the time varying parameters. For finite-time stability, Terminal SMC (TSM) has been designed in [25], which gives fast response, better accuracy and high robustness but faces slow convergence and singularity problem. To achieve quick convergence and remove singularity, Non- singular TSM (NTSM) and Non-singular Fast TSM (NFTSM) have been proposed to overcome these problems $[4,23]$. Furthermore, recent SMC methods integrated with notable control techniques have been proposed such as Adaptive SMC for uncertain interval type-2 Fuzzy systems with actuator saturation [12], output-feedback based Adaptive SMC for uncertain nonlinear Fuzzy systems [13], and model-free scheme using TSM to control attitude and position of uncertain quadrotor in the presence of external disturbances [20]. For robotic applications, there are few FTC methods that have been proposed, concern with the finite-time convergence. In [19], Non-Singular Fast Terminal Sliding Mode Control (NFTSMC) has been used to obtain rapid convergence and improvement in chattering and singularity problem, while time delay estimation (TDE) based FTC has been utilized for estimation of uncertainties and actuator faults. Since, the TDE is developed by inserting the delay in control method, which may deteriorate the performance of the system. To avoid the delay in the system, adaptive technique is preferred to compensate the uncertainties and faults. Adaptive control has exceptional capabilities to accommodate system uncertainties, external disturbances and component failures [18]. In fact, various adaptive schemes have been proposed to design FTC for a number of robotic applications to compensate actuator faults [10, 17]. Moreover, with NFTSMC scheme, adaptive control has been proposed for spacecraft attitude tracking in the presence of 
actuator faults, actuator saturations, disturbances and inertia uncertainties [7].

Fractional-order (FO) controller is extensively used to enhance the trajectory tracking and to guarantee the better control performance for both integer-order (IO) and FO systems [8]. FO controller with TSM and NTSM has been exploited in $[3,22]$, respectively. Moreover, model-free technique using Continuous Fractional-order Nonsingular Terminal Sliding Mode Control (CFONTSM) based on TDE has been proposed for practical tracking of robotic manipulators [21]. In [15], a remarkable technique named Adaptive Fractional-order Non-singular Fast Terminal Sliding Mode Control (AFONTSM) is proposed for tracking of robotic manipulator. Non-singular fast TSM is considered for fast convergence and singularity problem, while adaptive control approach is designed for estimation of disturbance and uncertainties, and FO is used to obtain good tracking performance of the system.

Thus, in this paper, we are focused upon investigating the Fractional-order Non-singular Fast Terminal SMC (FONFTSM) based FTC (FONFTSM- FTC) for uncertain robotic manipulators subject to the actuator failures. The main contributions of this work can be summarized as follows: 1) Sliding surface based on the properties of Fractional-order and Non-singular Fast Terminal SMC is developed, which gives rapid tracking performance, fast time convergence and mitigates the chattering problem in the control torque inputs of robotic manipulators. 2) FONFTSM-FTC is proposed using Adaptive technique; in which unknown uncertainties, external load disturbance and actuator faults are compensated to make the system reliable. 3) The system asymptotical stability is analyzed by Lyapunov theorem.

The remainder of this paper is organized as follows: Section-2 presents definition and lemmas about Fractional-order calculus. Section-3 describes the system modeling and problem formulations. Details of the control design and stability of the closed-loop system using Lyapunov analysis are presented in Section-4 and Section-5, respectively. Then in Section-6, comparative simulation results are provided to validate and demonstrate the effectiveness of the proposed approach. In the end, Section-7 concludes this paper.

Notations: The following notations in this work will be used such as $\|\cdot\|,|\cdot|$ and $\operatorname{sgn}(x)$ denote Euclidean norm, absolute value and sign function, respectively. $M$ is positive definite matrix, $0<\lambda_{\min }(M) \leq\|M\| \leq \lambda_{\max }(M)$, in which $\lambda_{\min }$ and $\lambda_{\max }$ illustrate the minimum and the maximum eigenvalues of matrix $M$. The superscript $T$ represents the transpose. The asterisk * sign will be used as element-wise multiplication between two vectors.

\section{Preliminaries}

Definition [11]: The Riemann-Liouville fractional integral and derivative of $\alpha_{t h}$-order with respect to $t$ and terminal value $a$ are defined as follow:

$$
\begin{aligned}
& { }_{a} \mathcal{I}_{t}^{\alpha} f(t)=\frac{1}{\Gamma(\alpha)} \int_{a}^{t} \frac{f(\tau)}{(t-\tau)^{1-\alpha}} d \tau \\
& { }_{a} \mathcal{D}_{t}^{\alpha} f(t)=\frac{d^{\alpha} f(t)}{d t^{\alpha}}=\frac{1}{\Gamma(1-\alpha)} \frac{d}{d t} \int_{a}^{t} \frac{f(\tau)}{(t-\tau)^{\alpha}} d \tau
\end{aligned}
$$

with function $f(t), \quad m-1<\alpha<m, \quad m \in \mathbb{N}$, $\alpha \in \mathbb{R}^{+}$and $\Gamma(\cdot)$ Gamma function, while $\mathcal{I}^{\alpha}$ and $\mathcal{D}^{\alpha}$ denote the fractional integral and derivative, respectively.

Property [16]: $n_{t h}$ order derivative $\left(d^{n} / d t^{n}\right)$ of the fractional-order derivative operator ${ }_{a} \mathcal{D}_{t}^{\alpha} f(t)$ transforms as

$$
\frac{d^{n}}{d t^{n}}\left({ }_{a} \mathcal{D}_{t}^{\alpha} f(t)\right)={ }_{a} \mathcal{D}_{t}^{\alpha}\left(\frac{d^{n} f(t)}{d t^{n}}\right)={ }_{t} \mathcal{D}_{a}^{\alpha+n} f(t)
$$

Lemma 1 [14]: To let the non-autonomous FO system have an equilibrium point at $x=0$

$\mathcal{D}^{\alpha} x(t)=f(x, t)$

Let there exists a continuously differentiable and Lipschitz with respect to $x$ Lyapunov function $V(t, x)$ such that

$$
\begin{gathered}
W_{1}\|x\| \leq V(t, x) \leq W_{2}\|x\|, \\
\dot{V}(t, x) \leq-W_{3}\|x\| \\
W_{1}\|x\| \leq V(t, x) \leq W_{2}\|x\| \\
\mathcal{D}^{\alpha} V(t, x) \leq-W_{3}\|x\|
\end{gathered}
$$

where $\alpha \in(0,1)$ and $W_{i}(i=1,2,3)$ is a class-K function. Then the equilibrium point of (3) is asymptotically stable.

Lemma 2 [24]: For finite-time stability, Lyapunov function $V(x)$ with initial value $V_{0}$ is given as 
$\dot{V}(x)+\sigma_{1} V(x)+\sigma_{2} V(x)^{\psi} \leq 0$,

where $\sigma_{1}, \sigma_{2}>0$ and $0<\psi<1$, and the settling time denoting by $T_{s}$ can be computed as follows:

$$
T_{s} \leq \frac{1}{\sigma_{1}(1-\psi)} \ln \left(1+\frac{\sigma_{1} V_{0}^{1-\psi}}{\sigma_{2}}\right)
$$

\section{Dynamics of Robotic Manipulators and Problem Statement}

The dynamic equation of the robotic manipulator can be defined as follows [19]

$$
\begin{aligned}
\ddot{q} & =M^{-1}(q)(\tau-C(q, \dot{q}) \dot{q}-G(q) \\
& -F(\dot{q})-\mathcal{W})+\eta\left(t-T_{f}\right) \chi(q, \dot{q}, \tau)
\end{aligned}
$$

where $q, \dot{q}$ and $\ddot{q} \in \mathbb{R}^{n}$ are joint's position, velocity and acceleration respectively. $M(q) \in \mathbb{R}^{n \times n}$ represents the inertia matrix, $C(q, \dot{q}) \in \mathbb{R}^{n \times n}$ denotes the coriolis and centripetal forces, $F(\dot{q}) \in \mathbb{R}^{n}$ represents the friction forces, $G(q) \in \mathbb{R}^{n}$ is the gravitational matrix, $\mathcal{W} \in \mathbb{R}^{n}$ expresses the unknown external load disturbance vector, $\tau \in \mathbb{R}^{n}$ stands for the joint torque vector, $\chi(q, \dot{q}, \tau) \in \mathbb{R}^{n}$ defines the partial and time-varying actuator fault vector, and $\eta\left(t-T_{f}\right)$ denotes the time profile of faults occurs at time $T_{f}$.

Moreover, the time profile of the above faults $\eta(\cdot)$ is defined as follows:

$$
\begin{aligned}
\eta\left(t-T_{f}\right)= & \operatorname{diag}\left\{\eta_{1}\left(t-T_{f}\right),\right. \\
& \left.\eta_{2}\left(t-T_{f}\right), \cdots, \eta_{n}\left(t-T_{f}\right)\right\},
\end{aligned}
$$

where $\eta_{i}$ denotes the $i_{t h}$ state equation affecting by the fault, and its corresponding fault model of time profile is given as follows:

$$
\eta_{i}\left(t-T_{f}\right)=\left\{\begin{array}{cc}
0 & \text { if } t<T_{f} \\
1-e^{-S_{i}}\left(t-T_{f}\right) & \text { if } t \geq T_{f}
\end{array},\right.
$$

where $\varsigma_{i}>0$ is the evolution rate of the unknown fault. When the value of $\varsigma_{i}$ is small, the fault is called an incipient fault. As $\varsigma_{i} \rightarrow \infty, \eta_{i}$ develops as a step function and the incipient fault becomes an abrupt fault.
At the occurring of an actuator fault $\vartheta \tau(t)$, the control torque $\tau(t)$ can be represented as [2]

$\tau(t)=\tau_{0}(t)+\vartheta \tau(t)$,

where $\tau_{0}$ is the nominal torque. The fault function $\chi(q, \dot{q}, \tau)$ in (8) can be expressed in the form of actuator fault $\vartheta \tau(t)$ as $\chi(q, \dot{q}, \tau)=M^{-1}(q) \vartheta \tau(t)$. Dynamic equation (8) can be rewritten as

$\ddot{q}=M^{-1}(q) \tau+f(q, \dot{q})+\Theta(q, \dot{q}, \tau)+\chi(q, \dot{q}, \tau),($

where $\quad f(q, \dot{q})=-M^{-1}(q)[C(q, \dot{q}) \dot{q}+G(q)]$ denotes the known nominal system dynamics and $\Theta(q, \dot{q}, \tau)=-M^{-1}(q)[F(\dot{q})+\mathcal{W}]$ expresses the uncertainties in the robotic manipulator.

For trajectory tracking, we can write (12) in the following form of tracking error

$\ddot{\tilde{q}}=M^{-1} \tau+f(q, \dot{q})+\Theta(q, \dot{q}, \tau)-\ddot{q}_{d}+\chi(q, \dot{q}, \tau)$,

where $\tilde{q}=q-q_{d}, q$ is actual and $q_{d}$ is desired position vectors.

\section{Fractional-order Non-singular Fast Terminal Sliding Mode Control based FTC}

In this section, sliding surface is, firstly, developed on the properties of Fractional-order and Nonsingular Fast Terminal Sliding Mode Control (FONFTSM). Subsequently, Control and Adaptive laws are designed to propose FONFTSM based FTC (FONFTSM-FTC) for uncertain robotic manipulators in the presence of partial and timevarying actuator faults at different joints.

\subsection{Sliding Surface Design}

To acquire benefits of TSM and FO control, and avoid the singularity problem, sliding surface has been designed in $[15,19]$. Motivated by the aforementioned techniques, accurate and rapid control performance for an $n$-degree of freedom ( $n$-DOF) manipulators in finite-time, the following referred FONFTSM surface can be selected as

$S(t)=\mathcal{D}^{\alpha} \dot{\tilde{q}}(t)+k_{1} \tilde{q}(t)^{\varphi}+k_{2} \tilde{q}(t)^{l / q}$,

where $S(t) \in \mathbb{R}^{n}$ is the sliding surface, $\alpha$ is FO between $0<\alpha<1, k_{1} \in \mathbb{R}^{n \times n}$ and $k_{2} \in \mathbb{R}^{n \times n}$ are 
positive constant diagonal matrices, while $l$ and $q$ are positive odd integers fulfilling the relation $1<l / q<2$ and $\varphi>l / q$.

To get the guarantee that sliding motion occurs, $\dot{S}(t)=0$ should be satisfied. According to the property (2), the derivative of (14) can be computed as

$$
\begin{aligned}
\dot{S}(t) & =\mathcal{D}^{\alpha} \ddot{\tilde{q}}(t)+k_{1} \varphi|\tilde{q}(t)|^{\varphi-1} * \dot{\tilde{q}}(t) \\
& +k_{2} \frac{l}{q}|\tilde{q}(t)|^{(l-q) / q} * \dot{\tilde{q}}(t)
\end{aligned}
$$

Since sliding manifold is developed, now FONFTSM-FTC control design will be considered for $n$-DOF manipulators to obtain robustness and reliability subject to unknown bounds of uncertainties and actuator faults.

Assumption: The bounded conditions of the uncertainties $\Theta(q, \dot{q}, \tau)$ and the fault vectors $\chi(q, \dot{q}, \tau)$ are given in (16) and (17), respectively, by the following functions

$$
\begin{gathered}
\|\Theta(q, \dot{q}, \tau)\| \leq\left(\beta_{0}+\beta_{1}\|q\|\right)\|S\|, \\
\|\chi(q, \dot{q}, \tau)\| \leq \beta_{\chi}(\|S\|+\|q\|\|S\|),
\end{gathered}
$$

where $\beta_{0}$ and $\beta_{1}$ are unknown constants of uncertainties upper bound, and $\beta_{\chi}$ is unknown constant denoting the upper bound of faults.

\subsection{FONFTSM-FTC Control Design}

The FONFTSM-FTC control law for robotic manipulator in presence of uncertainties, external load disturbance and actuator faults can be defined as follows:

$$
\tau(t)=\tau_{\text {nom }}(t)+\tau_{f}(t),
$$

where $\tau_{\text {nom }}(t)$ is the torque used to control nominal part and $\tau_{f}(t)$ is the control input utilized to compensate unknown uncertainties and actuator faults in the system. Their corresponding definitions are derived as follows:

$$
\begin{aligned}
\tau_{\text {nот }}(t) & =-M(q) f(q, \dot{q})+M(q) \ddot{q}_{d} \\
& -\mathcal{D}^{-\alpha}\{M(q) K S(t) *|S| \\
& +M(q) \operatorname{Ksgn}(S)^{\mu} *|S| \\
& +M(q) k_{1} \varphi|\tilde{q}(t)|^{\varphi-1} * \dot{\tilde{q}}(t) \\
& \left.+k_{2} \frac{l}{q} M(q)|\tilde{q}(t)|^{(l-q) / q} * \dot{\tilde{q}}(t)\right\}
\end{aligned}
$$

$$
\begin{aligned}
\tau_{f}(t) & =-\mathcal{D}^{-\alpha}\left\{M(q)\left(\hat{\beta}_{0}+\beta_{1}\|q\|\right) S(t)\right. \\
& \left.+M(q)\left(\hat{\beta}_{\chi}+\beta_{\chi}\|q\|\right) S(t)\right\}
\end{aligned}
$$

where $K=\operatorname{diag}\left(k_{1}^{\prime}, k_{2}^{\prime}, \cdots k_{n}^{\prime}\right)$ is a positive matrix, $\mu$ is a positive constant with range between $0<\mu<1$, and $\hat{\beta}_{0}, \hat{\beta}_{1}$ and $\hat{\beta}_{\chi}$ represent the estimates of $\beta_{0}, \beta_{1}$ and $\beta_{\chi}$, respectively.

For compensation of uncertainties, adaptive laws are developed as follows:

$$
\left\{\begin{array}{l}
\dot{\hat{\beta}}_{0}=\Gamma_{0}\|S(t)\|^{2} \\
\dot{\hat{\beta}}_{1}=\Gamma_{1}\|S(t)\|^{2}\|q\|
\end{array}\right.
$$

where $\Gamma_{0}$ and $\Gamma_{1}$ are positive parameters.

Since (21) and (22) are designed to estimate the upper bounds of the uncertainties and the unknown external load disturbance as well, one more adaptation law is designed for FTC to estimate the bound of partial and time-varying actuator faults. The updating law can be chosen as follows:

$\dot{\hat{\beta}}_{\chi}=\Gamma_{\chi}\left(\|S(t)\|^{2}\|q\|+\|S(t)\|^{2}\right)$,

where $\Gamma_{\chi}$ is the positive adaptation gain, and $\hat{\beta}_{\chi}$ is the estimate of unknown constant $\beta_{\chi}$ under bounded condition of fault vector (17). Hence, the FONFTSM-FTC scheme formulates the trajectory tracking performance of the robotic manipulators with uncertainties and actuator faults.

Remark 1: The proposed approach with the sliding manifold (14), and the control input law (18) updated by the adaptation laws (21-23), when applied to the robotic manipulator model (12), implies the tracking error $\lim _{t \rightarrow \infty} \tilde{q}(t) \rightarrow 0$. The asymptotical stability of closed-loop system is given in the next section.

\section{FONFTSM-FTC Stability Analysis}

In this section, the stability analysis of the system (13) is established by applying the Lyapunov stability theorem. The proposed approach guarantees the system is asymptotically stable under uncertainties, external disturbances and actuator faults.

Theorem: Considering the defined robotic manipulator (12) which has existing uncertainties, 
external load disturbance, and actuator faults, with the designed sliding mode surface (14) and control law (18), the system trajectory of robotic manipulator converges in finite-time with the adaptive laws (21-23) and Assumption (16-17).

Proof: The Lyapunov function is proposed to select as follows:

$$
\begin{aligned}
V(t)= & 0.5 S(t)^{T} S(t)+0.5 \Gamma_{0}^{-1} \widehat{\beta}_{0}^{2} \\
& +0.5 \Gamma_{1}^{-1} \widehat{\beta}_{1}^{2}+0.5 \Gamma_{\chi}^{-1} \widehat{\beta}_{\chi}^{2},
\end{aligned}
$$

where $\hat{\beta}_{0}=\hat{\beta}_{0}-\beta_{0}, \hat{\beta}_{1}=\beta_{1}-\beta_{1}, \hat{\beta}_{\chi}=\beta_{\chi}-\beta_{\chi} \quad$ are adaptation errors. By taking time derivative of $V(t)$, one gets

$$
\begin{aligned}
\dot{V}(t) & =S(t)^{T} \dot{S}(t)+\Gamma_{0}^{-1} \widehat{\beta}_{0} \dot{\hat{\beta}}_{0}+\Gamma_{1}^{-1} \widehat{\beta}_{1} \dot{\beta}_{1}, \\
& +\Gamma_{\chi}^{-1} \widehat{\beta}_{\chi} \dot{\hat{\beta}}_{\chi}
\end{aligned}
$$

To clarify the solution of (25), $\dot{S}(t)$ can be written in the simplified form by substituting (13) into (15), one has

$$
\begin{aligned}
\dot{S}(t) & =\mathcal{D}^{\alpha}\left\{M^{-1} \tau+f(q, \dot{q})+\Theta(q, \dot{q}, \tau)\right. \\
& \left.-\ddot{q}_{d}+\chi(q, \dot{q}, \tau)\right\}+k_{1} \varphi|\tilde{q}(t)|^{\varphi-1} * \dot{\tilde{q}}(t) \\
& +k_{2} \frac{l}{q}|\tilde{q}(t)|^{(l-q) / q} * \dot{\tilde{q}}(t)
\end{aligned}
$$

Then substituting (18) into the above equation (26), one obtains

$$
\begin{aligned}
\dot{S}(t)= & \mathcal{D}^{\alpha}\left\{M ^ { - 1 } \left[-M(q) f(q, \dot{q})+M(q) \ddot{q}_{d}\right.\right. \\
& -\mathcal{D}^{-\alpha}(M(q) K S(t) *|S| \\
& +M(q) K s g n(S)^{\mu} *|S| \\
& +M(q) k_{1} \varphi|\tilde{q}(t)|^{\varphi-1} * \dot{\tilde{q}}(t) \\
& +k_{2}(l / q) M(q)|\tilde{q}(t)|^{(l-q) / q} * \dot{\tilde{q}}(t) \\
& +M(q)\left(\hat{\beta}_{0}+\beta_{1}\|q\|\right) S(t) \\
& \left.\left.+M(q)\left(\hat{\beta}_{\chi}+\beta_{\chi}\|q\|\right) S(t)\right)\right] \\
& +f(q, \dot{q})+\Theta(q, \dot{q}, \tau)-\ddot{q}_{d} \\
& +\chi(q, \dot{q}, \tau)\}+k_{1} \varphi|\tilde{q}(t)|^{\varphi-1} * \dot{\tilde{q}}(t) \\
& +k_{2}(l / q)|\tilde{q}(t)|^{(l-q) / q} * \dot{\tilde{q}}(t)
\end{aligned}
$$

which can simplify as follows:

$$
\begin{aligned}
\dot{S}(t) & =-K S(t) *|S|-K \operatorname{sgn}(S)^{\mu} *|S| \\
& -\left(\hat{\beta}_{0}+\beta_{1}\|q\|\right) S(t) \\
& -\left(\hat{\beta}_{\chi}+\beta_{\chi}\|q\|\right) S(t) \\
& +\mathcal{D}^{\alpha} \Theta(q, \dot{q}, \tau)+\mathcal{D}^{\alpha} \chi(q, \dot{q}, \tau)
\end{aligned}
$$

By substituting (28) into (25), the following derivative of Lyapunov function $V(t)$ can be formulated as

$$
\begin{aligned}
\dot{V}(t) & =S(t)^{T}\left\{-K S(t) *|S|-K \operatorname{sgn}(S)^{\mu} *|S|\right. \\
& -\left(\hat{\beta}_{0}+\beta_{1}\|q\|\right) S(t)-\left(\beta_{\chi}+\beta_{\chi}\|q\|\right) S(t) \\
& \left.+\mathcal{D}^{\alpha} \Theta(q, \dot{q}, \tau)+\mathcal{D}^{\alpha} \chi(q, \dot{q}, \tau)\right\} \\
& +\Gamma_{0}^{-1} \widehat{\beta}_{0} \dot{\hat{\beta}}_{0}+\Gamma_{1}^{-1} \widehat{\beta}_{1} \dot{\beta}_{1}+\Gamma_{\chi}^{-1} \widehat{\beta}_{\chi} \dot{\beta}_{\chi}
\end{aligned}
$$

Moreover, with (21-23) in (29), one has

$$
\begin{aligned}
\dot{V}(t)= & -S(t)^{T} K S(t) *|S| \\
& -S(t)^{T} K \operatorname{sgn} S(t)^{\mu} *|S| \\
& -S(t)^{T}\left(\hat{\beta}_{0}+\beta_{1}\|q\|\right) S(t) \\
& -S(t)^{T}\left(\hat{\beta}_{\chi}+\beta_{\chi}\|q\|\right) S(t) \\
& +S(t)^{T} \mathcal{D}^{\alpha} \Theta(q, \dot{q}, \tau)+S(t)^{T} \mathcal{D}^{\alpha} \chi(q, \dot{q}, \tau) \\
& +\left(\hat{\beta}_{0}-\beta_{0}\right)\|S(t)\|^{2}+\left(\beta_{1}-\beta_{1}\right)\|S(t)\|^{2}\|q\| \\
& +\left(\hat{\beta}_{\chi}-\beta_{\chi}\right)\left(\|S(t)\|^{2}\|q\|+\|S(t)\|^{2}\right)
\end{aligned}
$$

With the introduction of $S^{T} S=\|S\|^{2}$ and $S^{T} \operatorname{sgn}(S)=\|S\|$, the above equation (30) can be deduced as

$$
\begin{aligned}
\dot{V}(t) & \leq-K\|S(t)\|^{2}\|S(t)\|-K\|S(t)\|\|S(t)\| \\
& -\|\left(\hat{\beta}_{0}+\beta_{1}\|q\|\|\|\|S(t)\|^{2}\right. \\
& -\left\|\left(\hat{\beta}_{\chi}+\beta_{\chi}\|q\|\right)\right\|\|S(t)\|^{2} \\
& +\|\Theta(q, \dot{q}, \tau)\|\|S(t)\|+\|\chi(q, \dot{q}, \tau)\|\|S(t)\| \\
& +\left\|\left(\hat{\beta}_{0}-\beta_{0}\right)\right\|\|S(t)\|^{2}+\left\|\left(\beta_{1}-\beta_{1}\right)\right\|\|S(t)\|^{2}\|q\| \\
& +\left\|\left(\hat{\beta}_{\chi}-\beta_{\chi}\right)\right\|\left(\|S(t)\|^{2}\|q\|+\|S(t)\|^{2}\right)
\end{aligned}
$$

Then according to Assumption (16-17), the above equation (31) can be simplified as follows

$$
\dot{V}(t) \leq-K\left(\|S(t)\|^{2}+\|S(t)\|\right)\|S(t)\|
$$

As we know $K>0$, then the trajectory tracking error system (13) of robotic manipulator is asymptotically stable. Thus the stability proof is analyzed completely.

Now, if we want to calculate its stability settling time, the above equation (32) can be rewritten as 


$$
\begin{aligned}
\dot{V}(t) \leq & -S(t)^{T} K S(t)\|S(t)\| \\
- & S(t)^{T} K \operatorname{sgn} S(t)^{\mu}\|S(t)\| \\
\leq & -2\|S(t)\| \lambda_{\min }(K)\left(\frac{1}{2} S(t)^{T} S(t)\right) \\
- & 2^{(\mu+1) / 2}\|S(t)\| \lambda_{\min }(K) \\
& \times\left(\frac{1}{2} S(t)^{T} S(t)\right)^{(\mu+1) / 2}
\end{aligned}
$$

in which $\lambda_{\min }(K)$ denotes the minimum eigenvalue of $K$.

$$
\begin{aligned}
\dot{V}(t) & \leq-2\|S(t)\| \lambda_{\min }(K)\left(1-\frac{Z}{V}\right) V \\
& -2^{(\mu+1) / 2}\|S(t)\| \lambda_{\min }(K) \\
& \times\left(1-\left(\frac{Z}{V}\right)^{(\mu+1) / 2}\right) V^{(\mu+1) / 2}
\end{aligned}
$$

with $Z=\frac{1}{2} \Gamma_{0}^{-1} \widehat{\beta}_{0}^{2}+\frac{1}{2} \Gamma_{1}^{-1} \widehat{\beta}_{1}^{2}+\frac{1}{2} \Gamma_{\chi}^{-1} \widehat{\beta}_{\chi}^{2}$.

Therefore, according to Lemma 2, the referred settling time can be computed as follows:

$$
T_{s} \leq \frac{2}{\Upsilon_{1}(1-\mu)} \ln \left(1+\frac{\Upsilon_{1} V_{0}^{(1-\mu) / 2}}{\Upsilon_{2}}\right)
$$

with

$$
\begin{aligned}
& \Upsilon_{1}=2\|S(t)\| \lambda_{\min }(K)\left(1-\frac{Z}{V}\right) \\
& \Upsilon_{2}=2^{(\mu+1) / 2}\|S(t)\| \lambda_{\min }(K)\left(1-\left(\frac{Z}{V}\right)^{\frac{(\mu+1)}{2}}\right)
\end{aligned}
$$

Hence, the error will converge to zero in a finitetime and the trajectory will maintain converging to the FONFTSM surface when $\lambda_{\min }(K)>0$.

Remark 2: From (18) and (35), one can observe that the $K$ is proportional to the control input $\tau$ and inversely proportional to the settling time $T_{\mathrm{s}}$. So, the suitable value of $K$ should be selected to obtain stable closed-loop system and finite settling time.

\section{Numerical Simulation Results}

In this section, to validate the proposed FONFTSM-FTC method, an uncertain 3-DOF robotic manipulator with unknown external load disturbance and its desired trajectories are discussed firstly. Secondly, an appropriate value of FO is selected by trail-and-error method for the developed FONFTSM-FTC without the actuator faults. Then, compared simulations on 2-DOF robotic manipulator without actuator faults are realized with a AFONTSM [15]. Finally, to validate the proposed method in the presence of actuator faults, compared simulations is implemented with a NFTSMC based active FTC (NFTSMC-FTC) [19].

\subsection{3-DOF Robotic Manipulator}

The referred 3-DOF robotic manipulator is three joints of PUMA 560 robot which is illustrated in [1]. Their initial joint positions are chosen as $q_{1}(0)=q_{2}(0)=q_{3}(0)=-\pi / 16$ and their corresponding desired trajectory tracking references, uncertainties and external load disturbances are selected respectively as follows:

$$
\begin{aligned}
& q_{d}=\left[\begin{array}{c}
\cos (t / 5 \pi)-1 \\
\cos (t / 5 \pi+\pi / 2) \\
\sin (t / 5 \pi+\pi / 2)-1
\end{array}\right], \quad \mathcal{W}=\left[\begin{array}{c}
0.5 \sin \left(\dot{q}_{1}\right) \\
1.1 \sin \left(\dot{q}_{2}\right) \\
0.15 \sin \left(\dot{q}_{3}\right)
\end{array}\right], \\
& F(\dot{q})=\left[\begin{array}{c}
0.5 \dot{q}_{1}+\sin \left(3 q_{1}\right) \\
1.3 \dot{q}_{2}-1.8 \sin \left(2 q_{2}\right) \\
-1.8 \dot{q}_{3}-2 \sin \left(q_{3}\right)
\end{array}\right] .
\end{aligned}
$$

\subsection{FONFTSM-FTC performance under different values of $\alpha$ without actuator faults}

In order to find a suitable FO value, the parameters of developed FONFTSM-FTC are selected as follows: for sliding surface (14), $k_{1}=90 \times \operatorname{diag}(20,20,20), \quad k_{2}=20 \times \operatorname{diag}(10,10,10)$, $l / q=1.05$ and $\varphi=1.5$, for control input (18), $K=\operatorname{diag}(10,10,10)$, and $\mu=0.9$ is selected to reduce chattering in control input, while adaptation gains and initial conditions of adaptation laws (21-23) are chosen as $\Gamma_{0}=\Gamma_{1}=\Gamma_{\chi}=5$ and $\hat{\beta}_{0}(0)=\beta_{1}(0)=\beta_{\chi}(0)=1$.

Since $\alpha \in(0,1)$, the performance of the proposed FONFTSM-FTC at different FOs without actuator faults are tested by the trial-and-error method on the referred uncertain 3-DOF robotic manipulator under unknown external load disturbance. Three different FO values of $\alpha=0.1, \alpha=0.3$ and $\alpha=0.6$ are selected, and their corresponding results of position tracking error and control 
input performances of FONFTSM-FTC are demonstrated in Figures 1-2, respectively.

From these obtained results, one can notice easily that the relative best performance result is obtained under $\alpha=0.1$ and it ensures the closed-loop system stability with satisfactory performance under uncertainties and unknown external load disturbances. Thus for the following comparison test, the suitable value of $\alpha=0.1$ which represents the fast convergent speed is selected. In addition, to illustrate the effectiveness of the proposed method, performance of position tracking and error at different initial joint as $q_{1}(0)=-\pi / 12$, $q_{2}(0)=-\pi / 9$ and $q_{3}(0)=\pi / 12$ are depicted at $\alpha=0.1$ in Figures 3-4, respectively.

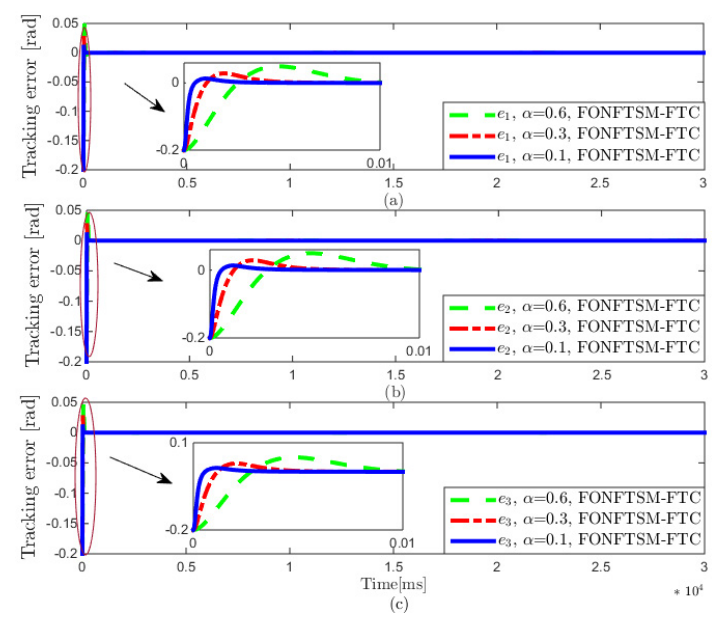

Figure 1. Tracking error performances under different $\alpha$ values. (a) Joint 1, (b) Joint 2 and (c) Joint 3
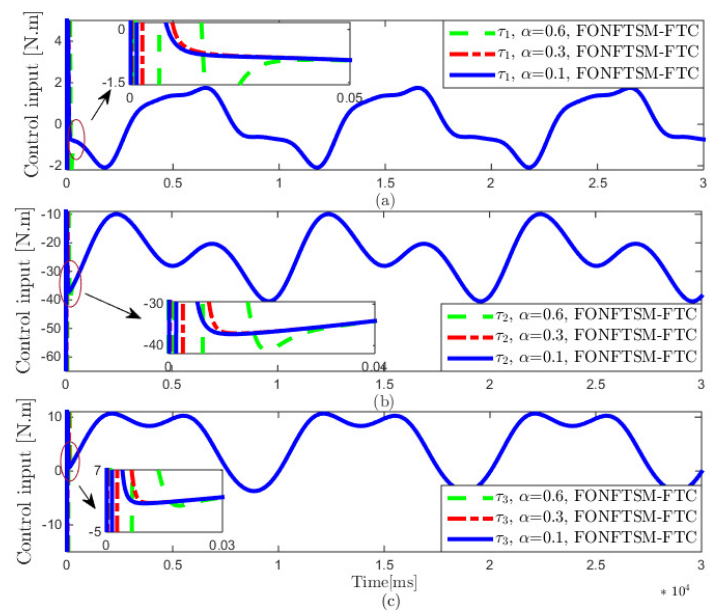

Figure 2. Control input performances under different $\alpha$ values. (a) Joint 1, (b) Joint 2 and (c) Joint 3
6.3 Comparison between FONFTSM-FTC and AFONTSM using 2-DOF Manipulator

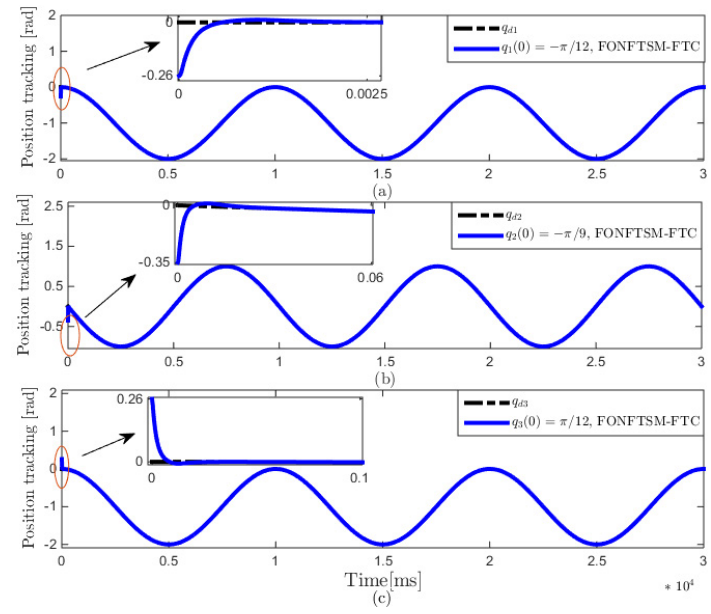

Figure 3. Joint's position tracking performances at different initial conditions under $\alpha=0.1$. (a) Joint 1 , (b) Joint 2 and (c) Joint 3

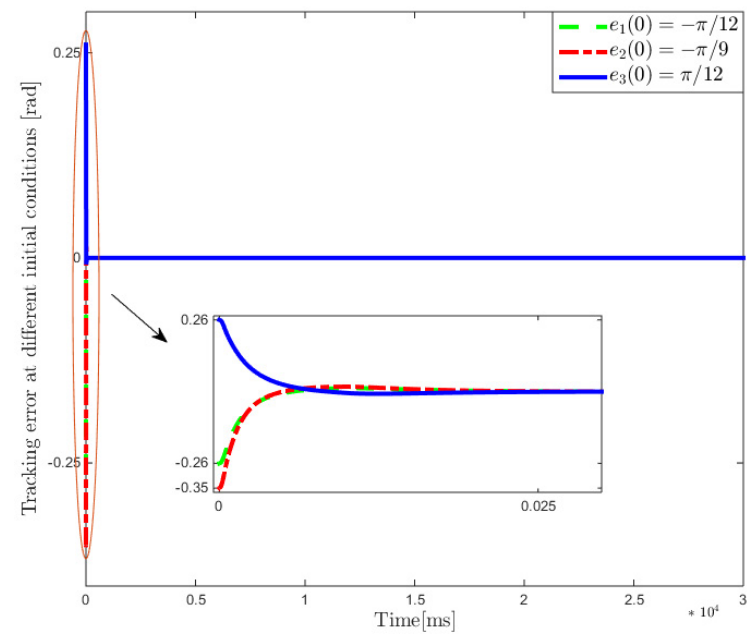

Figure 4. Tracking error performances at different initial conditions under $\alpha=0.1$

In this subsection, a 2-DOF robotic manipulator model has been used with uncertainties and external disturbances. In order to demonstrate the performance of FONFTSM-FTC, one realizes the comparison with the AFONTSM developed in [15]. Their model parameters, desired trajectories and uncertainties are chosen as same as described in [15]. Moreover, the other parameters of FONFTSM-FTC are selected as $\varphi=1.05$ and $l / q=1.01$. Thus, their corresponding simulation results are presented in Figures 5-6, which illustrate respectively the performances of position tracking and tracking errors. 
From these results, one can realize that the proposed FONFTSM has better performances than the compared AFONTSM in terms of fast tracking performance and small tracking errors.
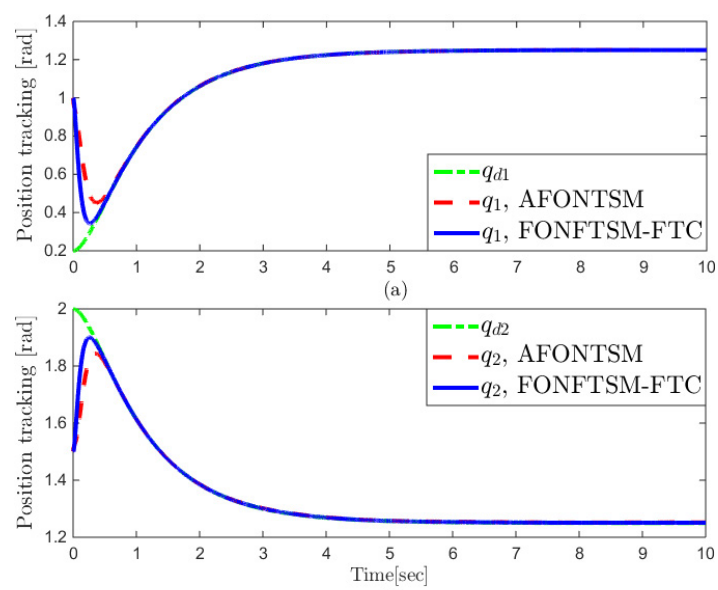

(b)

Figure 5. Comparison of position tracking between FONFTSM-FTC and AFONTSM. (a) Joint 1, (b) Joint 2
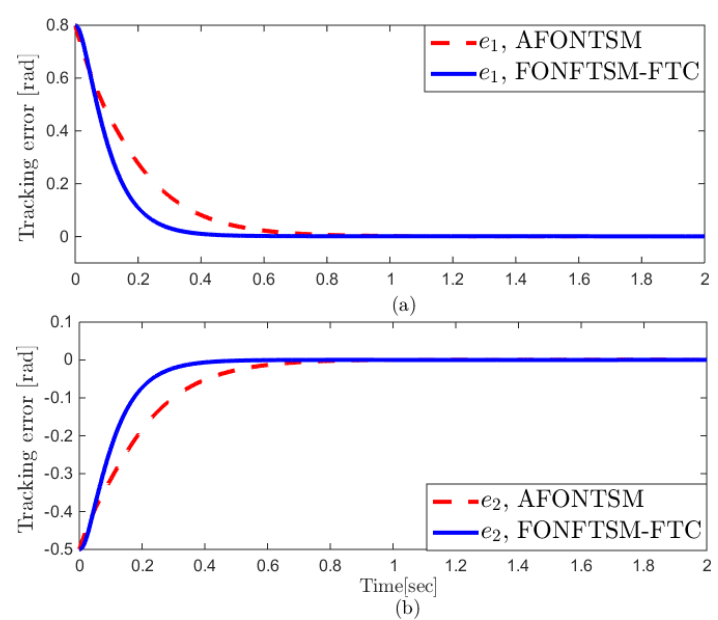

Figure 6. Comparison of tracking error between FONFTSM-FTC and AFONTSM. (a) Joint 1, (b) Joint 2

\subsection{Comparison between FONFTSM-FTC and NFTSMC-FTC with actuator faults}

Once selecting the best value of $\alpha=0.1$ for FONFTSM-FTC, then the considered fault vector is chosen as:

$$
\vartheta \tau_{1}=\left[0,20 q_{2}^{2}+15 \dot{q}_{2}^{2}+25 \cos \left(q_{2}\right)(10 s), 0.75 \tau_{3}(20 s)\right]^{T}
$$

which means that the faults $\vartheta \tau_{1}$ are applied respectively at joint 2 at $10 \mathrm{sec}$, and at joint 3 with $75 \%$ partial loss at $20 \mathrm{sec}$. Moreover, to demonstrate the proposed FONFTSM-FTC performance, one compares it with NFTSMCFTC [19]. And their corresponding results are illustrated in Figures 7-9, which depicts the joint's

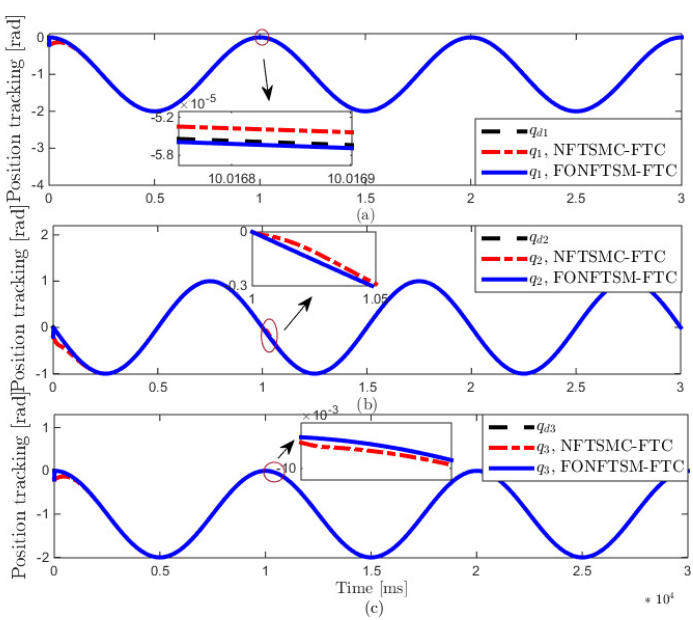

Figure 7. Comparison of position tracking between FONFTSM-FTC and NFTSMC-FTC under $\vartheta \tau_{1}$. (a) Joint 1, (b) Joint 2 and (c) Joint 3
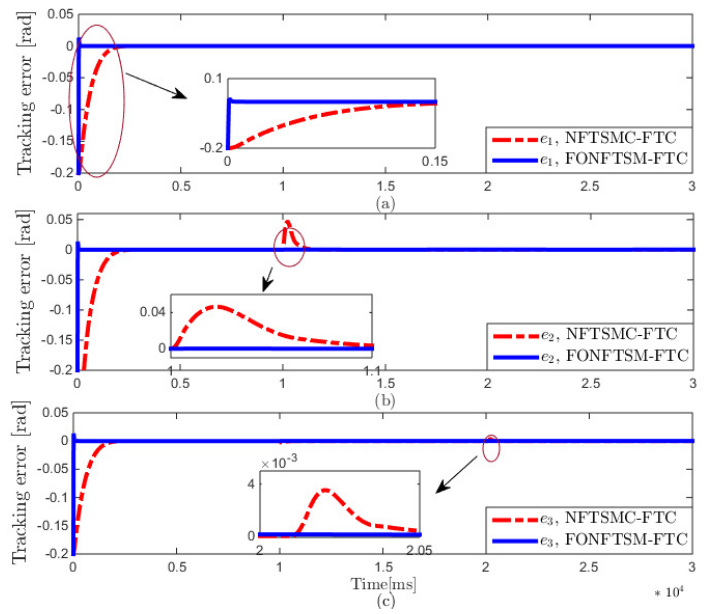

Figure 8. Comparison of tracking error between FONFTSM-FTC and NFTSMC-FTC under $\vartheta \tau_{1}$. (a) Joint 1, (b) Joint 2 and (c) Joint 3
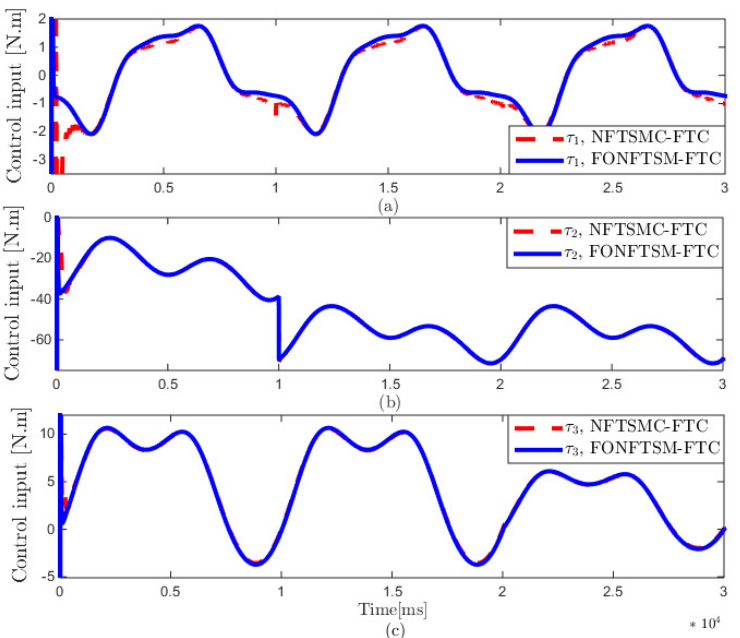

Figure 9. Comparison of control input between FONFTSM-FTC and NFTSMC-FTC under $\vartheta \tau_{1}$. (a) Joint 1, (b) Joint 2 and (c) Joint 3 
positions tracking, position tracking errors and control input signals, respectively.

From these results, one can notice that the proposed FONFTSM-FTC has better performances in terms of fast response speed, smaller tracking error and better compensation performance to actuator faults.

\section{Conclusion}

A FONFTSM-FTC is developed for trajectory tracking of a robotic manipulator in presence of uncertainties, unknown external load disturbance and actuator faults. Tracking performance and

\section{REFERENCES}

1. Armstrong, B., Khatib, O. \& Burdick, J. (1986). The Explicit Dynamic Model And Inertial Parameters Of The Puma 560 Arm. In Proceedings of the IEEE International Conference On Robotics And Automation (pp. 510-518).

2. Capisani, L. M., Ferrara, A., De Loza, A. F. \& Fridman, L. M. (2012). Manipulator Fault Diagnosis via Higher Order Sliding-Mode Observers, IEEE Transactions on Industrial Electronics, 59(10), 3979-3986.

3. Dadras, S.\& Momeni, H. R. (2012). Fractional Terminal Sliding Mode Control Design for a Class of Dynamical Systems With Uncertainty, Communications in Nonlinear Science and Numerical Simulation, 17(1), 367-377.

4. Feng, Y., Yu, X. \& Man, Z. (2002). NonSingular Terminal Sliding Mode Control Of Rigid Manipulators, Automatica, 38(12), 2159-2167.

5. Ghous, I. \& Xiang, Z. (2015). Reliable $\mathrm{H} \infty$ Control Of 2-D Continuous Nonlinear Systems With Time Varying Delays, Journal of the Franklin Institute, 352(12), 5758-5778.

6. Hagh, Y. S., Asl, R. M. \& Cocquempot, V. (2017). A Hybrid Robust Fault Tolerant Control Based on Adaptive Joint Unscented Kalman Filter, ISA Transactions, 66, 262-274.

7. Han, Z., Zhang, K., Yang, T. \& Zhang, M. (2016). Spacecraft Fault-Tolerant Control Using Adaptive Non-Singular Fast Terminal Sliding Mode, IET Control Theory \& Applications, 10(16), 1991-1999. fast finite-time convergence are achieved by FONFTSM, while FONFTSM-FTC is developed using Adaptive technique integrated with FONFTSM to compensate the uncertainties, unknown external load disturbance, and actuator faults. To demonstrate the performance of the proposed FONFTSM-FTC, compared simulation results are realized with AFONTSM on 2-DOF without actuator faults, and with NFTSMC-FTC on 3-DOF manipulator under actuator faults, and their corresponding results show that the proposed method has better performance under uncertainties, unknown external load disturbance and actuator faults.

8. Hua, C., Zhang, T., Li, Y. \& Guan, X. (2016). Robust Output Feedback Control For Fractional Order Nonlinear Systems With Time-Varying Delays, IEEE/CAA Journal of Automatica Sinica, 3(4), 477-482.

9. Kara, T. \& Mary, A. H. (2017). Adaptive Pd-Smc For Nonlinear Robotic Manipulator Tracking Control, Studies in Informatics and Control, 26(1), 49-58.

10. Kececi, E. F., Tang, X. \& Tao, G. (2009). Adaptive Actuator Failure Compensation For Redundant Manipulators, Robotica, 27(1), 19-28.

11. Li, C. \& Deng, W. (2007). Remarks on Fractional Derivatives, Applied Mathematics and Computation, 187(2), 777-784.

12. Li, H., Wang, J., Lam, H.-K., Zhou, Q. \& Du, H. (2016). Adaptive Sliding Mode Control for Interval Type-2 Fuzzy Systems, IEEE Transactions on Systems, Man, and Cybernetics: Systems, 46(12), 1654-1663.

13. Li, H., Wang, J. \& Shi, P. (2016). OutputFeedback Based Sliding Mode Control for Fuzzy Systems with Actuator Saturation, IEEE Transactions on Fuzzy Systems, 24(6), 1282-1293.

14. Li, Y., Chen, Y. \& Podlubny, I. (2010). Stability Of Fractional-Order Nonlinear Dynamic Systems: Lyapunov Direct Method and Generalized Mittag-Leffler Stability, Computers \& Mathematics with Applications, 59(5), 1810-1821.

15. Nojavanzadeh, D. \& Badamchizadeh, M. (2016). Adaptive Fractional-Order NonSingular Fast Terminal Sliding Mode Control 
for Robot Manipulators, IET Control Theory \& Applications, 10(13), 1565-1572.

16. Podlubny, I. (1998). Fractional Differential Equations: An Introduction to Fractional Derivatives, Fractional Differential Equations, to Methods of Their Solution and Some of Their Applications. Academic Press.

17. Rugthum, T. \& Tao, G. (2015). An Adaptive Actuator Failure Compensation Scheme For A Cooperative Manipulator System With Parameter Uncertainties. In IEEE Conference On Decision And Control (pp. 6282-6287).

18. Tao, G. (2014). Multivariable Adaptive Control: A Survey, Automatica, 50(11), 2737-2764.

19. Van, M., Ge, S. S. \& Ren, H. (2017). Finite Time Fault Tolerant Control For Robot Manipulators Using Time Delay Estimation And Continuous Nonsingular Fast Terminal Sliding Mode Control, IEEE Transactions on Cybernetics, 47(7), 1681-1693.

20. Wang, H., Ye, X., Tian, Y., Zheng, G. \& Christov, N. (2016). Model-Free-Based Terminal Smc Of Quadrotor Attitude And Position, IEEE Transactions on Aerospace And Electronic Systems, 52(5), 2519-2528.
21. Wang, Y., Gu, L., Xu, Y. \& Cao, X. (2016). Practical Tracking Control Of Robot Manipulators With Continuous FractionalOrder Nonsingular Terminal Sliding Mode, IEEE Transactions on Industrial Electronics, 63(10), 6194 - 6204.

22. Wang, Y., Luo, G., Gu, L. \& Li, X. (2015). Fractional-Order Nonsingular Terminal Sliding Mode Control Of Hydraulic Manipulators Using Time Delay Estimation, Journal of Vibration And Control, 1077546315569518.

23. Yang, L. \& Yang, J. (2011). Nonsingular Fast Terminal Sliding Mode Control For Nonlinear Dynamical Systems, International Journal of Robust and Nonlinear Control, 21(16), 1865-1879.

24. Yu, S., Yu, X., Shirinzadeh, B. \& Man, Z. (2005). Continuous Finite-Time Control For Robotic Manipulators with Terminal Sliding Mode, Automatica, 41(11), 1957-1964.

25. Zhao, D., Li, S. \& Gao, F. (2009). A New Terminal Sliding Mode Control for Robotic Manipulators, International Journal of Control, 82(10), 1804-1813. 